\title{
CATALOGUE
}

\section{RECENT SHELLS,}

NOW EXHEITING

\section{AT THE LONG ROOM, IN THE ROTUNDA, \\ COLLECTED BY}

THE LATE HENRY CHARLES SIRR, ESQ.

FORMEIL

TOWN MAJOR OF THE OTTY OF DUBLIN.

LOWH MaJOR OH TH OTIY OF DUBLIN.

NAMED AND ARRANGED BY

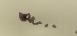

FREDERICK M`COY, EsQ., M.G.S., N.H.S.D., ETT.

\section{DUBLIN :}

GEORGE FOLDS, PRINTER, I, SAIN'T ANDREW-STREET, ARCHIVES (OPPQSITE TRINITY.STREET.) 


\section{CATALOGUE}

OF

\section{RECENT SHELLS,}

NOW EXHIBITING

\section{AT THE LONG ROOM, IN THE ROTUNDA,}

COLLECTED BY

THE LATE HENRY CHARLES SIRR, ESQ.

FORMERIY

TOWN MAJOR OF THE CITY OF DUBLIN.

NAMED AND ARRANGED BY

FREDERICK M‘COY, EsQ., M.G.s., N.H.S.D., ETC.

DUBLIN :

MUSEUM OF VICTORIA

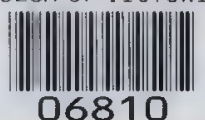

GEORGE FOLDS, PRINTER, 1, SAINT ANDREW-STREE'T,

(OPPOSITE THINITY_sTREET.) 



\section{P R E F C E.}

In drawing up the present Catalogue, of incomparably the finest collection of Shells in Ireland, I have endeavoured to lay before the public, in a tabular form, first, the name by which each species is known to the seientific of the prescnt day; next, the name by which it was known to Linné, or his followers; thirdly, the English name, or that by which it is linown, for the most part, to the dealers; and lastly, the country which the shell inhabits.

In so extensive a collcction, it of coursc happencd that some of the species had never before occurred to mc-those I hare noticed in the Appendix, where also I lave inscribed some notices from the New Fdition of Lamarck Animaux Sans Vertèbres, concerning the rarity of some of the little-known shells in the collection. I have not thought it necessary to dwell on those shells which have becn rendered famous by their scarcity-such, for instance, as the far-famed Conus cedo-nulli-the no-less valuable Orange Cowry, Scorpion Murex, Voluta fulgitrum, Glassy Nautilus, Wentle traps, \&c.-nor the host of others to be found here, whose names are familiar to every one from the extravagant prices they havo brought in the market.

F. $\mathrm{M} \cdot \mathrm{C}$. 
CONIA

20 porosa

porosa

porous

Kurile Islands

BALANUS

21 porcatus

poreata

ridged

East Indies

22 Scoticus

Scoticus

Scotch

Antrim

23.27 fistulosus

elavata

club shaped

Dublin Bay

28 rugrosus

rugosa

rugged

Devonslirc

29 striatus

striatus

striated

Holland

80 radialus

radiatus

purple rayed

Unknown

3I -39 tintinabulum

tintinabulum

belI

Jamaica

PENTELASMIS

40 striatus

anserifera

sfriated

Dublin Bny

4I lævis

anatifera

duck

Ilowth

\section{ASPERGILLUM SERPULA}

42 agglutinans

agglutinans

TEREDO

gigantea

\section{WORM SHELL}

carrier

Unknown

SEPTARIA

43 arenaria

TEREDO

44 navalis

navalis

ship

Bottom of ships

\section{PHOLAS}

PHOLAS

PIERCER

45 dactyloides

dactylus

prickley

Antrim

40 costata

costata

ribbed

East Indies

47-48 candida

candida

white

Malahide

49-5I crispata

crispata

curled

Malahide

52 parrus

$53-54$ pusillus

little

England

pusillus (in wood) dwarf

Mediterranean 
Modern Name

Old Name

English Name

Locality

PHOLIDEA

55 papyracea

papyracea

paper

lingland

SOLEN

$56-57$ siliqua

58-59 vagina

60-61 truncata

62-63 pelucidus

ENSATELLA

64-66 europœa

SOLENOCURTUS

67 legumen

68 caribous

69-70 strigilatus

$71-74$ radiatus

SOLETELLINA

75 rostrata

MYA

76 truncata

77 arenaria

ANATINA

78 subrostrata

70-81 Spengleri

82 convexa

83-85 myali:
SOLEN

siliqua

vagina

truncata

pclucidus

cnsis

legumen

caribous

strigilatus

radiatus

diphos

MYA

truncata

arenaria

SOLEN

anatinus

Spengleri

MYA

convexa

declivis

\section{RAZOR}

pod

sheath

Dublin Bay

Dublin Bay

truncated American Sea:

pelucid

Dubliu Bay

sword

Dublin Bay

peascod

caribcan

strigilated

purple-rayed

violet

Indian Ocean

\section{GAPER}

abrupt

Malahide

sand

Dublin Bay

RAZOR

duck's bill Indian Ocean

Spengler's Unknown

GAPER

convex

Irelinnd

sloping

Dublin Bay 


\begin{tabular}{|c|c|c|c|}
\hline Modern IName: & old Name & English Name & Locality \\
\hline LUTRARIA & MACTRA & WEDGE & \\
\hline 86 solenoides & hians & gaping & lreland \\
\hline 87-88 elliptica & lutraria & large & Ireland \\
\hline \multicolumn{4}{|l|}{ MAGTRA } \\
\hline - 89 Campeachiensi & Campeachicusi & groved & Bay of Campeachy \\
\hline 90 gigantea & solidissima & thick & New York \\
\hline $91-92$ carinata & carinata & kecled & Mediterranean \\
\hline 93 stultorum & stultorum & rayed & Dublin Bay \\
\hline 94 var. Americani & var. American & Amcrican variety & Amcrica \\
\hline $95-96$ rotundata & rotundata & rounded & Unknown \\
\hline \multicolumn{4}{|l|}{$97-98$ (see notes) } \\
\hline \multicolumn{4}{|l|}{ A MPHIDESMA } \\
\hline 99 Listeri & Listeri & Listcrs & Malahide \\
\hline CORBULA & MYA & \multicolumn{2}{|l|}{ GAPER } \\
\hline $100-103$ nuclcus & inequivalvis & unequal ralved & Dublin Bay \\
\hline PANDORA & TELLINA & \multicolumn{2}{|l|}{ TELLEN } \\
\hline 104-106 rostrata & inequivalvis & uncqual valved & La Mancha \\
\hline SAXICAVA & MYTILUS & MUSSEL & 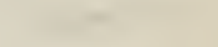 \\
\hline 107 rugosa & rugosus & rougl: & Dublin Bay \\
\hline SANGUINOLARIA & SOLEN & RAZOR & \\
\hline \multirow[t]{2}{*}{108 secidens } & occidens & spottcd & Ceylon \\
\hline & VENUS & VENUS & \\
\hline $109-117$ rugosa & deflorata & purple streaked & Mauritius \\
\hline PSAMMOBIA & TELLINA & TELLEN & \\
\hline 118 feriensis & ferónsis & Cornation & Dublin Bay \\
\hline
\end{tabular}




\section{7}

\begin{tabular}{lll} 
Modern Name & Old Name & English Name Locality \\
\hline SOLEN & RAZOR
\end{tabular}

119 vespertina vespertinus sun-set Galway

\section{TELLINA TELLINA TELLEN}

120-124 radiata radiata rayed Bahamas

125-127 unimaculata one spot Amimaculata

\begin{tabular}{|c|c|c|c|}
\hline 128-131 maculosa & interrupta & freckled & West India Islands \\
\hline $32-137$ virgata & virgata & striped & Ceylon \\
\hline
\end{tabular}

138 Madagascaricnsis Madagascariensis Madagascar Madagascar

$\begin{array}{rlll}\text { 139-140 rostrata } & \text { rostrata } & \text { beaked } & \text { Indian Occan } \\ \text { 141-142 latirostra } & \text { latirostra } & \text { broad beaked } & \text { Indian Ocean } \\ 143 \text { sulphurea } & \text { sulphurea } & \text { sulphur } & \text { Indian Ocean }\end{array}$

144 (see notes)

$\begin{array}{rlll}\text { 145-147 foliacea } & \text { foliacca } & \text { leafy } & \text { Indian Oecan } \\ 148 \text { depressa } & \text { depressa } & \text { depressed } & \text { Malahide } \\ \text { 149-151 rosea } & \text { rosea } & \text { rosy } & \text { Unknown } \\ 152-153 \text { fabula } & \text { fabula } & \text { semistriatcd } & \text { Dublin Bay } \\ 154-157 \text { tenuis } & \text { tenuis } & \text { thin } & \text { Dublin Bay } \\ 158-160 \text { donacina } & \text { donacina } & \text { donax } & \text { Dublin Bay } \\ 161 \text { scorbinata } & \text { scorbinata } & \text { rasp } & \text { Surat } \\ 162 \text { crassa } & \text { crassa } & \text { thick } & \text { Bray } \\ 163-166 \text { lingula felis } & \text { lingula felis } & \text { cats tongue } & \text { Nicobar lslands } \\ 167-176 \text { solidula } & \text { solidula } & \text { solid } & \text { Dublin Bay } \\ 177-178 \text { bimaculata } & \text { bimaculata } & \text { twin spot } & \text { Ircland }\end{array}$

$179-186$ (sce notes) 


\begin{tabular}{|c|c|c|c|}
\hline Modern Name & Old Name & English Name & Locality \\
\hline LUCINA & VENUS & VENUS & \\
\hline 187-189 Jamaicensis & Jamaicensis & Jamaica & Antilles \\
\hline 190-193 Pennsylvanica & Pennsylvanica & Pennsylvanian & America \\
\hline 194 edentula & cdentula & toothless & Jamaica \\
\hline $195-197$ radula & spuria & thread-girded & Dublin Bay \\
\hline 198-199 undata & undata & waved & Dublin Bay \\
\hline DONAX & DONAX & WEDGE & \\
\hline 200-203 scortum & scortum & beaked & East lndies \\
\hline 204 cuneata & cuneata & euneate & East Indies \\
\hline 205-207 denticulata & denticulata & toothed & Mediterranean \\
\hline \multirow[t]{2}{*}{ 208-209 trunculus } & trunculus & yellow & Dublin Bay \\
\hline & VENUS & VENUS & \\
\hline \multirow[t]{2}{*}{$210-221$ meroc } & meroe & merỏe & Indian Ocean \\
\hline & DONAX & WEDGE & \\
\hline 222-226 scripta & scripta & lettered & Senegal \\
\hline 227 castanea & castanca & chesnut & Unknown \\
\hline CRASSINA & VENUS & VENUS & \\
\hline 228 Scotica & Scotica & Scotch Venus & Dublin Bay \\
\hline 229 Danmoniensis & Danmoniensis & Devonshire & Eugland \\
\hline CYCLAS & TELLINA & TELLEN & \\
\hline 230 rivicola & cornea & River & England \\
\hline \multicolumn{4}{|l|}{231 (sec notes) } \\
\hline \multicolumn{4}{|l|}{ CYRENA } \\
\hline 232 Cayenensis & & & Batavia \\
\hline
\end{tabular}




\begin{tabular}{cccc} 
Modern Name & Old Name & English Name & Localit \\
\hline CXPRINA & VENUS & VENUS & \\
$233-234$ Islandica & Islandica & Iceland Venus & Ireland \\
$235-238$ chinensis & sinensis & Chinese & China
\end{tabular}

\section{CYTHEREA}

$239-244$ impudica
$245-246$ castanea
$247-248$ meretrix
$249-251$ morphina
$252-258$ (sec notes)
259 corbicula
$260-262$ petechialis
$263-269$ erycina
270 costata
$271-274$ chionc
275 (see notes)
CIRCE

276-278 maculata

279-283 castrensis

maculata

spotted

Brazil

castrensis

eastern

Amboyna

\section{CYTHEREA}

284-287 tigerina

289-293 dionc

294 (sce notes)

295ั-297 lineta

lincta

tiger

West Indies

dione

prickley mouthed South America

298 exoleta

299 concentrica

exoleta

concentrica
Dublin Bay

antiquated Dublin Bay

concentric Jamaica 


\begin{tabular}{|c|c|c|c|}
\hline Modern Name & Old Name & English Name & Locality \\
\hline 300 scripta & seripta & written & Amboyn: \\
\hline $301-304$ pectinata & pectinata & toothed & Amboyza \\
\hline $305-308$ flexuosa & flexuosa & Hexuous & West Indies \\
\hline \multicolumn{4}{|l|}{309 (see notes) } \\
\hline \multicolumn{4}{|l|}{ VENUS } \\
\hline $310-313$ puerpura & puerpura & spotted & China \\
\hline $314-316$ corbis & corbis & & Indian Ocean \\
\hline 317.318 verrucosa & verrucosa & warty & Dublin Bay \\
\hline $319-3 \supseteq 0$ rugosa & rugosa & rugged & East Indies \\
\hline 321 cassina & cassina & broad ribbed & Ireland \\
\hline $322-325$ granulata & granulata & granulated & West ludies \\
\hline $326-329$ mercenaria & mercenaria & Wanpum & Connecticut \\
\hline $330-331$ gallina & gallina & Hen & Dublin Bay \\
\hline 332 lamellata & lamellata & fringed & New Holland \\
\hline \multicolumn{4}{|l|}{$333-334$ (see notes) } \\
\hline 335-339 papilionacca & rotundata & butterfly's wing & Indian Ocean \\
\hline $340-341$ adspersa & litterata var. & dotted & Indian Occan \\
\hline 342-343 punctifera & punctata & lettered & Indian Ocean \\
\hline 344 litterata & litterata & lettered variety & Indian Ocean \\
\hline 345 litterata var. & nocturna & cloudy & Indian Ocean \\
\hline $346-347$ textile & textile & tissue & Red Sea \\
\hline 348-352 decussata & decussata & decussated & Malabar \\
\hline $353-354$ pullastra & pullastra & Sandwich & Dublin Bay \\
\hline $355-356$ fasciata & fasciata & thick ribbed & Dublin Bay \\
\hline 357-360 paphia & paphia & wrinkled & West Indies \\
\hline
\end{tabular}




\begin{tabular}{clll}
\hline Modern Name & Old Name & English Name & Locality \\
\hline 361 -368 cingenda & cingenda & girdled & Maryland \\
369 var. cingenda & cingenda var. & girdled & Unknown \\
$370-371$ thiara & thiara & turban & lndian Occan \\
372 caliste & caliste & sordid white & Red Sea \\
$373-380$ (see notes) & & & .
\end{tabular}

\section{CARDIUM}

$381-383$ costatum

384 ringens

885-387 echinatum

388-390 aculeatum

391 -393 medium

394-396 muricafum

397-400 muricatum?

$401-408$ isocardida

409 erinaceum

410 papyraccum

411 clongatum

412 magnum

413 ventricosum

414 sulcatum

415-417 serratum, Lam.

418-426 lævigatum, Lam.

427-428 biradiatum

429-432 rusticum

$433-434$ edule
CARDIUM

costatum

ringens

echinatum

aculeatum

medium

muricatum

muricatum var.

isocardida

spinosum

papyraceum

elongatum

magnum

maculatum

oblongum

lrvigatum, $\mathrm{Pcn}$.

scrratum

rusticum

edulc

\section{COCKLE}

ribbed

toothed

thorny

spiny

marbled

prickley

prickley

imbricated

hedgc-hog

paper

lengthened

giant

ventricosc

oblong

smooth

foreign, smooth Atlantic

rose spotted Indian Ocean

banded

common
Mcditcrranean

\section{Senegal}

Africa

Ireland

Ireland

lndia

West Indies

West Indies

Jamaica

Mediterranean

Indian Ocean

Antilles

Jamaica

Coast of Campeaehy

Mediterranean

Dublin Bay

Dublin Bay 


\begin{tabular}{|c|c|c|c|}
\hline Modern Name & Old Name & English Name & Locality \\
\hline 435 Grœnlandieum & Grœnlandieum & Greenland & Greenland \\
\hline 436 latum & latum & broad & Indian Ocean \\
\hline 437 crenulatum & crenulatum & small toothed & Ireland \\
\hline $438-439$ unedo & unedo & white strawberry & Amboyna \\
\hline $440-444$ retusum & retusum & Diana's heart & East Indies \\
\hline $445-446$ eardissa & cardissa & spiny-keeled & East Indies \\
\hline CARDITA & CHAMA & CLAMP SHELL & \\
\hline $447-451$ bieolor & bieolor & two eolored & Red Sea \\
\hline $452-453$ calyculata & ealyculata & variegated & Indian Seas \\
\hline 454 phrenetiea & semiorbieulaia & brown & Arabia \\
\hline 455 antiquata & antiquata & antiquated & Adriatie \\
\hline 456 Ajar & Ajar & Ajar & Mouth of the Niger \\
\hline \multicolumn{4}{|l|}{$457-458$ (see notes) } \\
\hline \multicolumn{4}{|l|}{ ISOCARDIA } \\
\hline $459-466$ cor & eor & heart & Dublin Bay \\
\hline 467-468 Moltkiana & Moltkiana & white grooved & New Holland \\
\hline CUCULLAA & $\mathrm{ARCA}$ & $\triangle \mathrm{RC}$ & \\
\hline 469-471 auriculifera & eueullus & chambered & Nicobar Island \\
\hline \multicolumn{4}{|l|}{$\mathrm{ARCA}$} \\
\hline $472-473$ tortuosa & tortuosa & twisted & Amboyna \\
\hline 474-477 Nore & Noæ & Noah's & Red Sea \\
\hline 478 tetragona & tetragona & four-sided & Mediterranean \\
\hline $479-480$ ventricosa & imbrieata & imbricated & Afriea \\
\hline 481 barbata & barbata & bearded & Mediterranean \\
\hline
\end{tabular}


482-484 antiquata

485-488 granosa

489 scnilis

490 (sce notes)

\section{PEETUNCULUS}

$491-492$ glycimeris

493-495 pilosus

496 seriptus

497-503 marmoratus

504-507 stellatus

508 violascescens

$\$ 09$ pectiniformis

510 pectunculus antiquata

granosa

senilis

glycimeris

pilosa

scripta

marmorata

stellata

violascens

pectunculus

pectinatus antiquated

Barbadoes

grained

Tranquebar

rugose

Jamaica

$511-514$ (unknown to Lamark) pectiniformis (Wood) scallop

\begin{tabular}{|c|c|}
\hline orbicular & Adriatic \\
\hline downy & Mediterranean \\
\hline written & St. Domingo \\
\hline marbled & Mediterranean \\
\hline white star & Africa \\
\hline violet & Mediterrancan \\
\hline pecten & Asiatic Ocean \\
\hline comb-like & West Indies \\
\hline
\end{tabular}

Unknown

\section{NUCULUS}

515 rostratus

rostrata

beaked

Baltic

\section{TRIGONIA}

516 pectinata

UNIO

517 hopetonensis

\section{THELIDERMA}

518 peruviana

519 pustulosa

\section{EGLIA}

520 ovata

\section{CHAMA}

toothed

New Holland

GAPER

Hopetown Darian

MYA

hopetonensis

Darian

$\begin{array}{lll}\text { peruviana } & \text { peruvian } & \text { Brazil } \\ \text { pustulosa } & \text { warted } & \text { Alabama }\end{array}$


Modern Name

old Name

English Name

Locality

$\operatorname{MYSC} \Lambda$

$521-523$ pietorum

pietorum

painters

England

POTOMIDA

524 geometriea

geometrica

geometrie

Bayouteche

$525-529$ sinuata

sinuata

notehed

Spain

530 cormgata

corrugata

wrinkled

India

\section{LYMNADIA}

531 alata

alata

winged

South Ameriea

HYRIA

532 avicularis

syrmatophora

angular

Brazil

\section{MYCETOPUS}

$533-534$ solenoides

solenoides

solen-like

Unknown

SYMPHYNOTA

535 Woodiana

Woodiana

Woods

China

536 magnifiea

magnifica

magnifieent

China

\section{ALASMODON}

537-540 margaritifcra

margaritifera

pearl

Ireland

MARGARITANA

541 deltoidea

deltoidea

triangular

Cincinati

UNIOPSIS

$542-543$ pyramidatis

pyramidatis

pyramidal

Ohio

544 mytiloides

mytiloides

mussel-like

Ohio

545-547 (in another case)

\section{ANODON}

$548-551$ sygneus

sygneus

swan

Grand Canal

552 anatinus

anatinus

duek

Royal Canal 


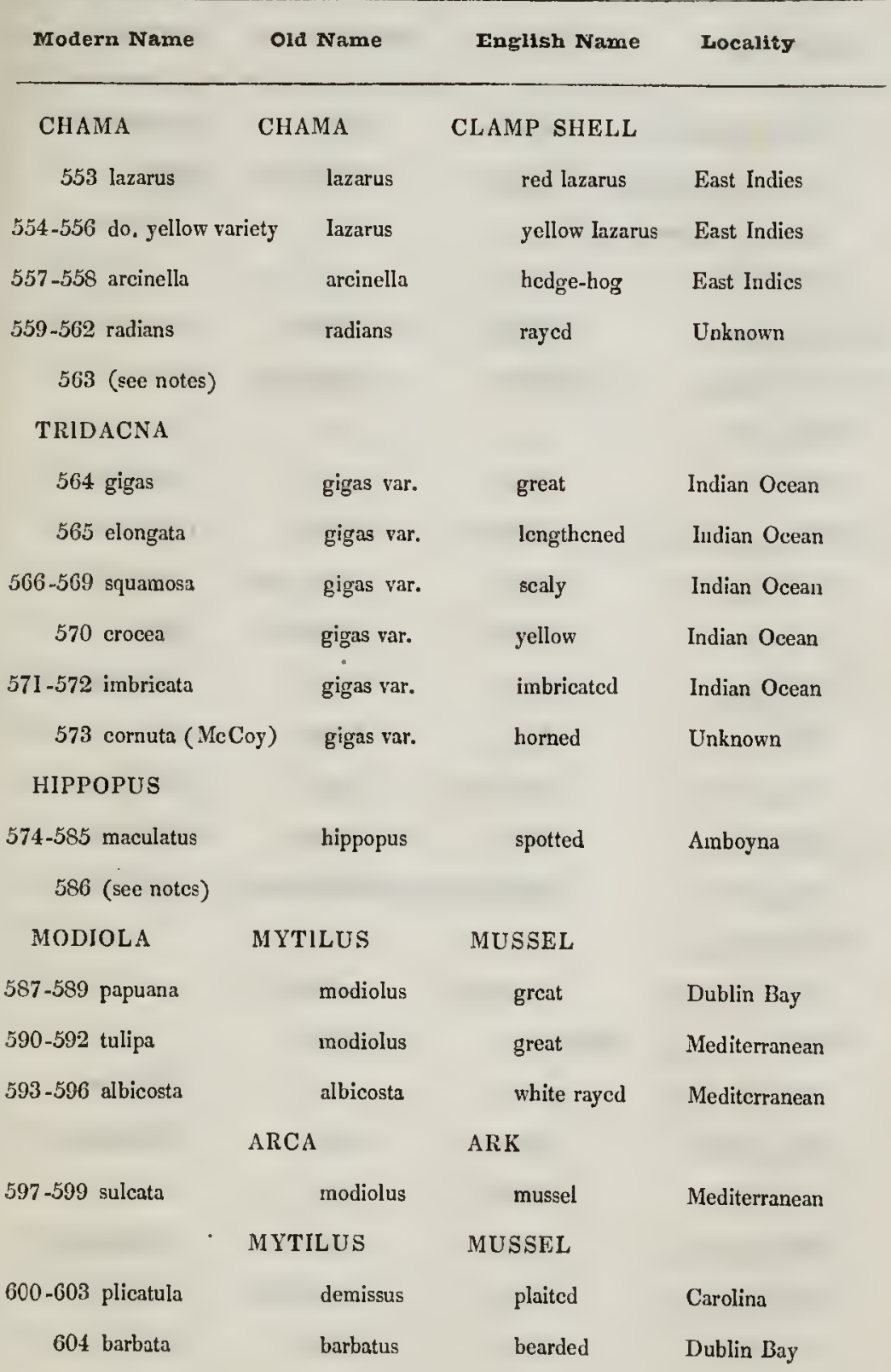




\section{Modern Name}

Old Name

English Name

Iocality

$\begin{array}{llll}605 \text { discors, (Lam.) } & \text { impactus } & \text { woolly } & \text { New Holland } \\ 606 \text { discrepans (Lam.) } & \text { diserepans } & \text { compartment } & \text { France } \\ 607-609 \text { (see notes) } & & & \end{array}$

\section{LITHODOMUS}

\author{
610-617 dactylus \\ 618-620 caudigerus
}

\section{MYTILUS}

\begin{tabular}{|c|c|c|c|}
\hline 621 opalus & opalus & opal & Tranguebar \\
\hline $622-625$ smaragdinus & smaragdinus & emerald & Trangushia \\
\hline 626 Magellanieus & bidens & double tooth & America \\
\hline 627 crenatus & erenatus & toothed & Carolina \\
\hline 628 latus & latus & wide & Van Dieman's Land \\
\hline 629 afer & afer & Barbary & Barbary \\
\hline 630 acatinus & latus (Pars.) & jasper & New Holland \\
\hline $631-633$ edule & cdule & common & Dublin Bay \\
\hline 634 edule & \multicolumn{2}{|c|}{ cdule (singular reparation of injury) } & Dublin Bay \\
\hline \multicolumn{4}{|l|}{ DRESSINA } \\
\hline 635 polymorpha & polymorpha & mottled & \multirow{2}{*}{ Russia } \\
\hline PINNA & PINNA & NACKER & \\
\hline 636 rudis & rudis & rough & West Indies \\
\hline 637 squamosa & squamosa & scaly & Mediterrancan \\
\hline 638 seminuda & rigida & rigid & Curaçoa \\
\hline 639 pectinata & pectinata & spiny-ribbed & Mediterranean \\
\hline 640 exusta & adusta & pcar-shaped & Manilla \\
\hline 641 nigra & nigra & black & Red Sea \\
\hline
\end{tabular}

$\begin{array}{lll}\text { lithophagus } & \text { burrowing } & \text { West Indies } \\ \text { aristatus } & \text { cross-beaked } & \text { Africa }\end{array}$


642 ingens

643 dolabrata

644 saccata

PERNA

645-647 ephippium

648 isognomum

649 (see notes)

\section{MALLEUA}

650 albus

65 I -653 vulgaris

\section{AVICULA}

654-655 hirundo

656-657 (see notes)

MELEAGRINA

658-664 margaritefera

665-666 albina

$667-668$ radiata

\section{LIMA}

$669-670$ fragilis

671-674 squamosa

$675-676$ glacialis

\section{PECTEN}

677 maximus

678 Jacobœus

679 medius ingens

bicolor

saccata

OSTREA

ephippium

isognomum

albus

malleus

MYTILUS

hirundo

margaritifera
albina
radiata

OSTREA

fragilis

lima

glacialis

$\operatorname{maxima}$

Jacoboa

medius great Devonshire

two-coloured Red Sea

satchel-shaped Indian Ocean

OYSTER

saddle Pulo Condora

long hinged Luconia

white hammer New Holland

hammer Tranquebar

MUSSEL

swallow

Mediterranean

Persian Gulf

New Holland

Tranquebar

$\begin{array}{ll}\text { brittle } & \text { Bray } \\ \text { file } & \text { Meditcrranean } \\ \text { icey } & \text { West Indies }\end{array}$

SCALLOP

great

Scotland

lesser

Mediterrancan

middle 


\section{8}

\begin{tabular}{|c|c|c|c|}
\hline Madern Name & Old Name & English Name & Locality \\
\hline $680-684$ zie-zic & zie-zic & zig-zig & Providence \\
\hline 685-686 Japonicus & Japonica & Japan & Japan \\
\hline 687 Laurentia & Laurentia & Lorenzo's & South Seas \\
\hline 688.689 pleuronectes & pleuronectes & sole & Molueca Islands \\
\hline 690 radula & radula & rasp & Tranquebar \\
\hline 691 distans & distans & clouded-grey & Unknown \\
\hline 692 palliata & palliata & variegated & Mediterranean \\
\hline 693-699 nodosus & nodosa & knobbed & Senegal \\
\hline $700-701$ pallium & pallium & Duealmantle & India \\
\hline 702 plica & rosea & rosy & Red Sea \\
\hline 703-706 opereularis & opereularis & painted & Dublin Bay \\
\hline $707-708$ lincatus & lineatus & red-lined & Youghal \\
\hline 709 irradians & irradians & duskey & \\
\hline \multicolumn{4}{|l|}{$710-711$ (sce notes) } \\
\hline $712-713$ Islandicus & Islandiea & Iceland & Grecnland \\
\hline $714-717$ varius & varia & painted & Dublin Bay \\
\hline 718 sanguineus & sanguinea & searlet & West Indies \\
\hline 719 unicolor & unieolor & red & Unknown \\
\hline \multicolumn{4}{|l|}{720 (see notes) } \\
\hline HINNITES & - & 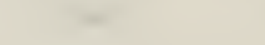 & 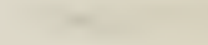 \\
\hline $721-723$ sinuosus & pusio & distorted & Dublin Bay \\
\hline SPONDYLUS & SPONDYLUS & IIINGED OYSTER & \\
\hline 726 gœdaropus & gœdaropus & red thorny & Mediterrancan \\
\hline 727-729 Amcricanus & goedaropus var. & American & St. Domingo \\
\hline 730 longispina & goedaropus var. & long spined & Unknown \\
\hline
\end{tabular}




\section{Modern Name}

Old Name

English Name

Locality

731 regius

732 spathuliferus

733 aurantits

OSTREA

734 edulis

735 Canadensis

736 gibbosa

OSTREA

regius

royal

India

gœdaropus var.

flat spined

Unknown

godaropus var.

China

OYSTER

edule

edible

Dublin Bay

Canadensis

Canada

New York

gibbosa

gibbose

Jamaica

\section{MYTILUS}

737-739 crista-galli

740-74I hyotis

742 radiata

743 mytiloides

744-748 rubella

VULSELLA

749 linguatula

PLACUNA

750-75I sella

752-753 placenta

754 papyracea

ANOMIA

755-757 ephippium

758 fornicata

TEREBRATULA

759 vitroea

760 rosea crista-galli

hyotis

radiatus

frons

MYA

vulsella

ANOMIA

sella

placenta

papyracea

cphippium

fornicata

vitroea

rosea
MUSSEL

cocks'-comb China

horned Friendly Islands

horned giant Indian Ocean

Indian Ocean

West Indies

GAPER

tongue shaped Tranquebar

\section{ANOMIA}

saddle

Java

moon China

paper saddle Red Sea

orbicular

Dublin Bay

arched

Unknown

glassy Mediterranean

rosy

New Holland 


\begin{tabular}{|c|c|c|c|}
\hline Modern Name & Old Name & English Name & Locality \\
\hline $761-762$ psittacea & psitfacea & parxot-beaked & Greenland \\
\hline 763 dorsata & dorsata & keeled & Straits of Magellan \\
\hline 764 dentata & dentata & toothed & Australia \\
\hline 765 flavescens & flavescens & yellowish & Java \\
\hline \multicolumn{4}{|l|}{ HYALCA } \\
\hline $766-767$ tridentata & tridentata & three-toothed & Mediterranean \\
\hline 768 (see notes) & & & Nile \\
\hline 769 (see notes) & & & Nile \\
\hline CHITON & CHITON & COAT OF MALL & \\
\hline 770 ruber & lube: & small red & Dublin Bay \\
\hline 771 castaneus & castaneus & chesnut & Unknown \\
\hline 772 squamosus & squamosus & scaly & Carolina \\
\hline 773 viridis & viridis & green & Unknown \\
\hline 774 piceus & piceus & pitchy & Red Sea \\
\hline 775 gigas & gigas & great & Cape of Good Hope \\
\hline 776 magnificus & magnificus & great black & Chili \\
\hline \multicolumn{4}{|l|}{ 777-778 (see notes) } \\
\hline \multicolumn{4}{|c|}{ PHAKELOPLEURA } \\
\hline 779 fascicularis & fascicularis & tufted & Dublin Bay \\
\hline \multicolumn{4}{|c|}{ CANTHAPLEURA } \\
\hline 780 spinosa & spinosus & spiny & South Sea \\
\hline PATELLA & PATELLA & LIMPET & \\
\hline $781-789$ granatina & granatina & garnet & Cape of Good Hope \\
\hline 790 oculus & oculus & goats eye & Island of Cyprus \\
\hline
\end{tabular}


791 tenuis

792-796 umbella

\section{LOTTTIA}

797-798 testudinaria

P $\triangle$ TELLA

799 eochlear

800 eompressa

801 (scc notes)

802-809 deaurata

$810-813$ vulgata

8I4-8]7 pelucida

818-821 Iævis

822-823 Magellanica

824 asper

825-828 monopis

829 mamillaris

830 repanda

831-832 plicata

833-838 tessellata

839-855 (see notcs)

356-858 virginca

PARMOPHORTS

859 australis

FISSUREILA

860 rosea tenuis

mineati

testudinaria

cochlear

compressa

deaurata

vulgata

pelucida

locvis

fusea

asper

monopis

mamilluris

repanda

plicata

tessellata

virginea

ambigua

nubecula thin

Unknown

rose-streaked Isle of Bourbou

fortoise seale Fast Indies

horse-shoc New Zealand

flat-sided Otalıeid:

gold-tipped Falkland Islands

common Dublin Ray

b]ue rayed Irelind

smooth France

sugarwionf Magellan

rough Cuknowu

chesnut-strcak Unknown

nipple $\quad$ Africa

small sun

Island of Cerigo

plaited

Magellin

tessellated Norway

red-rayed

Norway

white dueks bill New Holland

variegated, red

Mediterranean 


\begin{tabular}{|c|c|c|c|}
\hline Modern Name & Old Name & English Name & Locality \\
\hline $861-862$ nimbosa & nimbosa & sealy, ribbed & Sencgal \\
\hline 803 crassa & crassa & thick & Unknown \\
\hline $864-865$ goea & groeca & cancellated & Dublin Bay \\
\hline \multicolumn{4}{|l|}{$866-867$ (see notes) } \\
\hline \multicolumn{4}{|l|}{ EMARGINUIAA } \\
\hline $868-870$ fissura & fissura & slit & Dublin Bay \\
\hline \multicolumn{4}{|l|}{ PLLEOPSIS } \\
\hline $871-873$ Ilungariea & Hungariea & Hungarian bonnet & t Dubliu Bar \\
\hline $874-875$ militaris & Inilitaris & honked & England \\
\hline \multicolumn{4}{|l|}{ CALYPTAA } \\
\hline $876-877$ extinctorum & extinctorum & extinguislier & Atlantic Oecan \\
\hline 878 Sinchsis & sinensis & Chinese bonnet & Dubliu Bay \\
\hline $879-881$ (see notes) & & & \\
\hline
\end{tabular}

\section{INFUNDIBULUM}

$$
882 \text { pileus }
$$

CREPIDUI, A

\begin{tabular}{|c|c|c|c|}
\hline 883-881 porcelau & porcellauus & brown-spolted & Indian Seus \\
\hline SCAPHANDER & B【せLLA & HIPPER & \\
\hline $885-887$ lignaria & liguaria & wood & Dublin Bay \\
\hline \multicolumn{4}{|l|}{ VESICA } \\
\hline $8 \& 8-591$ ampulla & amp̧ulla & pewits' egg & Amboyna \\
\hline $892-896$ natนeum & naucilum & sca-nut & Java \\
\hline $897-898$ physis & physis & striped & Enst Indies \\
\hline 899_901 amplustre & amplustre & bauded & China \\
\hline$\$ 02$ hydatis & hyclatis & paper & Ireland \\
\hline
\end{tabular}




\section{Nodern Name \\ Old Name \\ English Name \\ Locality}

\section{BULLQEA}

903_905 aperti

aperta

wide

Ircland

906 fragilis

akera

clastic

Dublin Bay

\section{HELIX}

907-908 pomatia

909 aspe:sa

910-913 licmastoma

914-921 inelimotragus

922-93I nemoralis

932 arbustorum

933-935 hortensis

936 monozonalis

937 lineolati

938 maticoides

939 contusa

240-943 Senegalensis

944 lactea

945-947 picta

948-966 (sec notes)

267-987 Carolinensis

988-992 (sce notes)

EPISTYLA

993-994 conicn

\section{ONITES}

995-997 (sce notes)
HELIX

pounatia

hortensis

lıcmastomi

melinotragus

nemoralis

arbustorum

monozalis

lineata

apertis

contusa

Senegalensis

lactea

picta

Carolinensis

rpisiylium
SNAIL

elible

lirance

garlen

Dublin

rose-lipped Panama

black-bordered Brazil

varied

Irclaux]

brown single streak Antrim

common Dublin

single band Unknown

lined

Amerie:

graping

lirunce

blunt-tipped Brazil

left handed Africa

milky

Isarbary

painted

Amboyı

Carolina

North America

bee hive

South Sea Islands 


\begin{tabular}{|c|c|c|c|}
\hline Modern Name & Old Name & Fnglish Name & Locality \\
\hline 998-1000 pisana & virgata & striped & Dublin \\
\hline $1001-103$ algira & algira & yellowish & Amboyna \\
\hline \multicolumn{4}{|l|}{ NANINIA } \\
\hline \multicolumn{3}{|l|}{ DISCODOMA } & India \\
\hline \multicolumn{4}{|l|}{$1008-1010$ (see notes) } \\
\hline 1011 gigas & lampas & orange lip & Unknown \\
\hline 1012 albilabris & carocalla & large brown & America \\
\hline 1013-1016 marginata & marginata & white margined & Jamaica \\
\hline 1017 inflata & inflata & inflated & Porto Rico \\
\hline 1018 Gualteriana & Gualteriana & Gualteri's & Spain \\
\hline \multicolumn{4}{|l|}{ LUCERNA } \\
\hline $1019-1020$ soror? & soror & four-toothed & West Indies \\
\hline 1021 sinnata & sinuata & sinuous & Barbadocs \\
\hline $1022-1025$ acutissima & acutissima & sharp edged & Jamaica \\
\hline \multicolumn{4}{|l|}{ LYROSTOMA } \\
\hline \multicolumn{4}{|l|}{ ANASTOMA } \\
\hline 1030 globulosa & globulosa & small grinner & Unknown \\
\hline PUPA & TURBO & WREATH SHELL & \\
\hline $1031-1033$ mumia & mumia & double toothed & America \\
\hline $1034-1035$ uva & uria & berry & Bretagny \\
\hline \multirow[t]{2}{*}{1036 muscorum } & muscorum & moss & Ireland \\
\hline & IIELIX & SNAlL & \\
\hline 1037 odontostuma & odontostuma & six toothed & Brdzil \\
\hline
\end{tabular}


Modern Name

\section{BULIMUS}

1038-1040 ovatus

Jô4 homastomus

1042-1044 melanostomus

I945 .1046 citrinus

1047 inversus

1048 lrvis

1049 decollatus

1050 - I061 (see notes)

\section{GIBB $\Lambda$}

1062-1064 Lyonettianus

\section{BULIMULUS}

1065 undatus

1066-1067 zic-zac

1068-1069 mexicanus

GONIOSTOMA

1070 erubescens

\section{ACIIATINA}

$1071-1079$ perdix

1080 Zebra

1081 immaculata

1082 bicarinata

\section{BULIMUS}

bicarinatus

\section{BULLA}

English Name Locality

Ceylon

Surinam

Panama

Guyana

India

party-colored East Indies

truncated Brazil
Lyonettianus distorted East Indies

\section{DIPPER}

waved

Antilles

zig-zig:

Unknown

Mexican

Mexico

\section{WREATI SHELL}

angle mouthed Brazil

\section{DIPPER}

broad striped Cape of Good Hope

Zebra Guinea

unspotted Unknown 


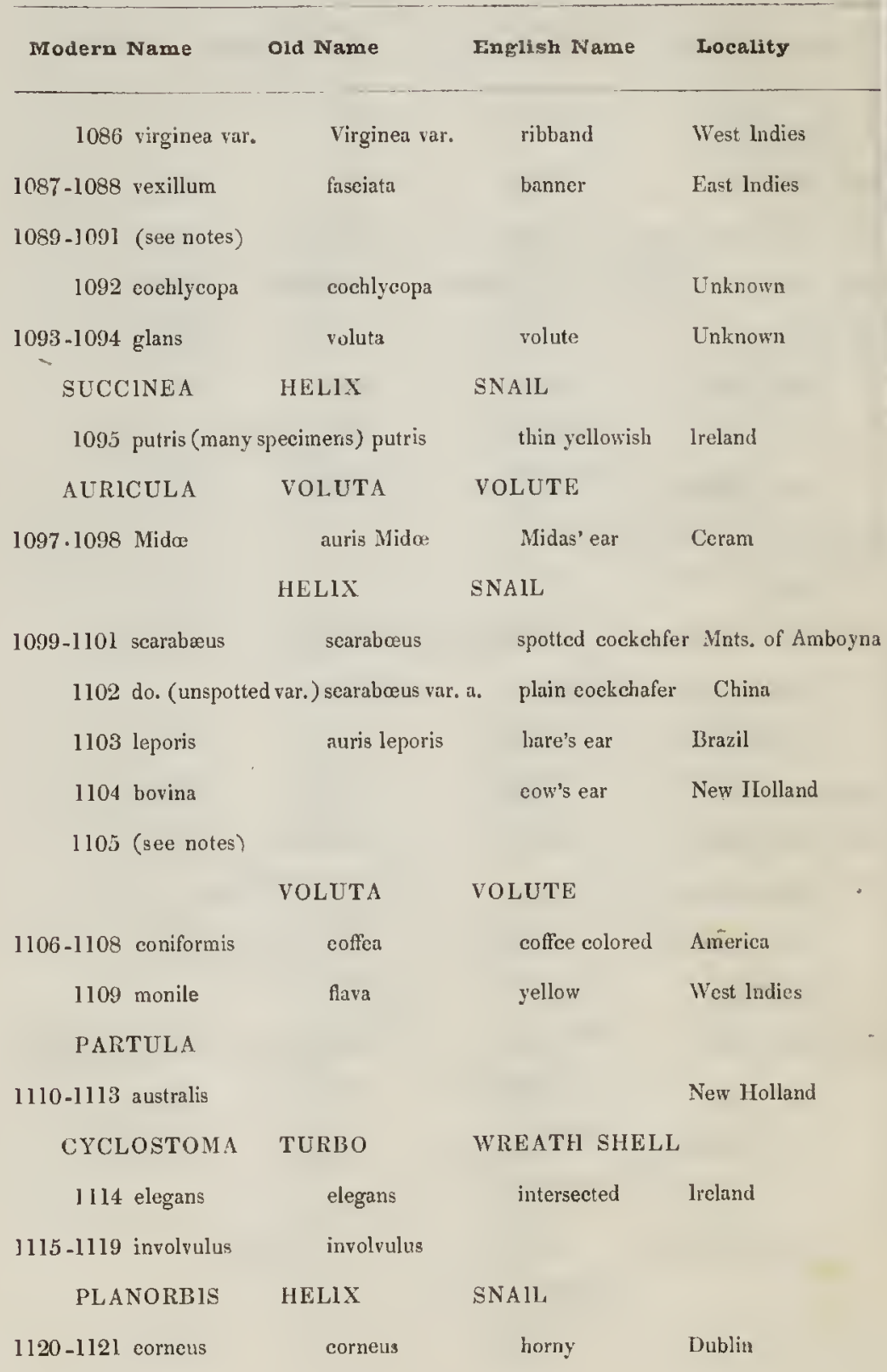


Modern Name old Name English Name Locality

1122 do., reversed

1123 cornu-arietis

1124 lutescens

1125 carinatus

LYMNEA

$1126-1128$ stagnalis

1129 auricularia

1130.1135 palustris

1136.1140 pereger

1141 columna

\section{PALUDINA}

1142 impura

1143 vivipara

MELAGANTHA

1144 amarula

MELANIA

1145 (see notes)

AMPULLARIA

1146 Guyanensis

1147 rugosa

1148-1149 effusa

1150 canaliculata

$1151-1153$ fasciata

1154-1156 (see notes) corneus

horny

Meath

cornu-arietis

rams horn

Brazil

lutescens

yellowish

Unknown

carinatus

keeled

Ireland

stagnalis

auricularia

palustris

pereger

columna

column

Dublin

lake

Bermingham

large eared

marsh

Dublin

Dublin

Guinea

$\begin{array}{lll}\text { tentaculata } & \text { olive } & \text { Dublin } \\ \text { vivipara } & \text { viviparous } & \text { England }\end{array}$

amarula

mitre

Madagascar

$\begin{array}{lll}\text { Guyanensis } & \text { striped } & \text { Demarara } \\ \text { urceus } & \text { cocoa-nut } & \text { Mississippi } \\ \text { glanca } & \text { greyish-brown } & \text { Antilles } \\ \text { canaliculata } & \text { chanelled } & \text { Guadaloupe } \\ \text { ampullacea } & \text { banded } & \text { Amboyna }\end{array}$


NERITINA

\begin{tabular}{|c|c|c|c|}
\hline $1157-1159$ pulligera & pulligera & reddish & Moluccas \\
\hline 1160 virginea & virginea & zig-zag & St. Domingo \\
\hline 1161 lacustris & lacustris & lake & ltaly \\
\hline \multicolumn{4}{|l|}{ CLITHON } \\
\hline 1162 corona & corona & Eastern Crown & Ganges \\
\hline \multicolumn{4}{|l|}{ NERITA } \\
\hline $1163-1169$ peloronta & peloronta & bloody tooth & Red Sea \\
\hline 1170 atrata & atrata & smoth black & Goree \\
\hline 1172-1180 polita & polita & mottled & Sand \\
\hline 1181 lactaria & lactaria & milky & Madagascar \\
\hline 1182 lineata & lincata & lined & Straits of Malacca \\
\hline 1183-1203 Ascensionis & Ascensionis & Aseension & lsland of Ascension \\
\hline $1204-1207$ versicolor & versicolor & purplc tessalated & Indian Ocean \\
\hline $1208-1215$ tessellata & tessellata & tessalated & Magdalen lslands \\
\hline 1216 Malaceensis & malaccensis & Malacca & Malacca \\
\hline \multicolumn{4}{|l|}{$1217-1218$ (see notes) } \\
\hline \multicolumn{4}{|l|}{ NALICA } \\
\hline $1219-1221$ glaucina & glaucina & livid & Mediterranean \\
\hline \multicolumn{4}{|l|}{$1222-1223$ (see notes) } \\
\hline 1224-1227 mamilla & mamilla & nipple & Cape of Good Hope \\
\hline $1228-1230$ monilifera & glaucina, var. & brown & Dublin Bay \\
\hline 1231-1232 Alderi & intricata & Alder's & Dublin Bay \\
\hline $1233-1235$ mamillaris & mamillaris & nipple & Antilles \\
\hline
\end{tabular}


$1236-1240$ vitcllus

1241-1:245 millepunctata

$1247-1255$ (sce notes)

$1256 \cdot 1259$ melanostomil

1:260-1263 anelsuoidens

IANTIIINA

1264-1260 communis

HAIIOTHS

1267-1270 tubiler.

$1271-1273$ iris

$1.274-1.275$ (see notes)

$1276-1277$ midio

1278-1279 Clinacherodii

1280-1282 asinia

$1283-1285$ australis

1:286-1297 tuberenlatit

1298 striata

1299 (see notes)

STOMA'TIA

1300 pliymotis

SIGARETUS

130I-1:302 haliotoillous

TOLNATELLA vitellus

stercus muscu'um clouded yellow

Bantam

llousand spot Aureriea
IIELIX

SNAIL

mamillaris

arachoidens

junthina

IIALIOTS

grigantea

iris

nidie

Cleracherodii

aม่เกiาม

anstralis

tubereulatia

striata

imperforntat

IIELIX

haliotoideus

VOLUT $\Lambda$

solidula

tornatilis

$$
\text { mitunillary }
$$

Guinea

spicler's wel

lnknown

violet

Meditertancin

\section{LAR SIIEL,}

giimst

New Ilolland

iris

New Zealliund

leaiy

Cilpen of Good llope

Chracherode's Calcfornia

ilss's call

Chint

rough phited New Zealand

(พ))แกาเง

$\Lambda$ driatic:

wrinliled

liallary
SNAlI.

venus' c'ar

Ireland
1303 soliclula

1304-1305 fasciata strongt

China

double banded Dubliu Bay 
PYRAMIDELLA

1306 dolabrata

VERMETUS

1307-1308 lumbricalis

SCALARIA

1309-131l pretiosa

1312-1313 Pallassii

1314-1316 Turtoni

1317-1318 Australis

1319 lamellosa

1320-1325 communis

IELPHINULA

1326-1330 laciniata

1331 distorta

SOLARIUM

1332-1343 perspectivum

ROTELLA

1344 linealata

1345 rosea

1346 Australis

TROCHUS

$1347-1354$ (see notes)

1355-1356 niloticus

1357-1359 pyramidalis

1360-1361 (sec notes)

$1362-1363$ imperialis
TROCHUS

dolobratus

SERPULA

lumbricalis

TURBO

scalaris

scalaris var.

Turtoni

Australis

lamellosa

clathratus

delphinus

distortus

TROCHUS

perspectivus

vestiarius

roseus

- Australis

niloticus

faveolatus

imperialis
TOP SHELL

zebra

Africa

WORM SHELL

flexuous

Goree

WR EATH SHELL

wentle trap Indian Ocean

small wentle trap Unknown

Turton's - Dublin Bay

southern New Holland

distant-ribbed Unknown

latticed

Mediterranean

cndive leaf

Amboyna

disjoined

Unknown

TOP SHELL

stair-case

Indian Occan

flattened

Mediterranean

rosy

Indian Ocean

southern

Indian Ocean large marbled Pulocondore

sugar loaf, Red Sea

imperial
New Zealand 
1364-1367 longissimus

1368 Mauritiana

1369-1373 fenestratus

1374-1377 spinosus

1378-1379 argyrostoma

1380-1384 agglutinaus

1385-1388 pellis-serpentis

1389-1391 selectus

1392 granatum

1393-1394 papillosus

1395-1398 ditto var.

1399-1404 ziziphinus

1405-1408 magus

1409-1414 iris

1415 dolarius

1416-1417 merula

1418-1419 maculatus

$1420-1424$ virgatus

$1425-1431$ cinerareus

1432-1433 Lyonsii

1434-1446 Pharonis

1447 elegans

1448-1450 cælatus

1451-1454 imbricatus

1455-1464 tuber
Mauritianus

alveare

spinosus

argyrostoma

conchiliophorus

pellis-serpentis

selectus

granatum

papillosus

papillosus

ziziphinus

magus

iris

dolarius

sinensis

maculatus

virgatus

cinerareus

Lyonsii

Pharonis

elegans

cælatus

imbricatus

tuber
Indian Ocean

New Guinea

Frederick 1sland

New Zealand

Arabia Felix

St. Domingo

Panama

red \& white grained New Zealand

tiger

New Zealand

granulated

Dublin Bay

forcign granulated East 1ndies

livid

Dublin Bay

tuberculated

Dublin Bay

iris

New Zealand

fluted

New Zealand

Chinese

China

spotted

Madagascar

rose-striped $\quad$ mboyna

greyish

Dublin Bay

white

Dublin Bay

strawberry

Red Sea

elegant

New Zealand

scaly

St. Croix

imbricated

Martinique

large mottled

\section{Barbadoes}




\begin{tabular}{|c|c|c|c|}
\hline Modern Name & Old Name & English Name & Locality \\
\hline \multicolumn{4}{|l|}{ MONODONTA } \\
\hline 1465-1470 labio & labio & double lipped & Manilla \\
\hline \multicolumn{4}{|l|}{ 1471-1475 (see notes) } \\
\hline $1476-1477$ rugosa & rugosus & rough & Unknown \\
\hline \multicolumn{4}{|l|}{$1478-1481$ (see notes) } \\
\hline & TURBO & WREATH SHELL & \\
\hline 1482-1484 pagodus & pagodus & pagoda & Moluccas \\
\hline \multicolumn{4}{|l|}{ TURBO } \\
\hline 1485-1486 pertholatus & pertholatus & serpents skins & Sumatra \\
\hline 1487-1488 smaraticus & smaraticus & large nobbed & False Bay \\
\hline $1489-1492$ olearius & olearius & large keeled & Coromandel \\
\hline 1493-1499 pica & pica & magpie & West Indies \\
\hline $1500-1501$ cornutus & cornutus & large horned & China \\
\hline $1502-1508$ setosus & setosus & leopard & Isle of France \\
\hline 1509 argyrostomus & argyrostomus & silver mouthed & Indian Ocean \\
\hline $1510-1511$ chrysostomus & chrysostomus & golden mouthed & Friendly lslands \\
\hline 1512 cochlus & cochlus & Camelopard & Trederick lslands \\
\hline 1513 smaragdus & smaragdus & green & East Indies \\
\hline 1514 versicolor & porphyrites & porphyry & New Caledonia \\
\hline 1515 eidaris & cidaris & turban & New Guinca \\
\hline $1516-1518$ rugosus & rugosus & large silver mouth & Languedoc , \\
\hline 1519 armatus & armatus & armed & Bay of Naples \\
\hline $1520-1521$ coronatus & coronatus & crown & Straits of Malacea \\
\hline $1522-1523$ margaritaccus & margaritaceus & pearly mouth & Frederick's Island \\
\hline $1524-1527$ muricatus & muricatus & prickley & Goree \\
\hline
\end{tabular}




\section{\begin{tabular}{l} 
Modern Name Old Name English Name Locality \\
\hline
\end{tabular}}

1528-1529 Moltkiana

moltkiana

moltkian

China

1530 (see notes)

PLANAXIS

$1531-1532$ sulcata

PHASIANELLA

1533-1538 bulimoides

TURRITELLA

1539-1540 duplicatus

1541-1544 ditto var.

1545-1554 terebra

1555-1557 (see notes)

1558-1559 fuscata

1560 Archimedes

1561 bicingulata

CERITHIUM

1562-1565 telescopium

$$
\begin{aligned}
& 1566-1567 \text { ebenium } \\
& 1568-1569 \text { muricatum } \\
& 1570-1572 \text { obeliscus } \\
& 1573-1575 \text { asperum } \\
& 1596 \text { lineatum } \\
& 1577-1578 \text { vertagus } \\
& 1579-1585 \text { fasciatus }
\end{aligned}
$$

\section{BUCCINUM WHELK}

sulcatum

grooved

Tranquebar

Australe phesant New Zealand

TURBO

duplicatus

ditto, var.

terebra

\section{WREATH SHELL}

double ribbed Mediterranean

double ribbed .Unknown

auger screw Dublin Bay

fuscata brown Unknown

Archimedes Archimedes' screw China

variegata

variegated

Spain

\section{TROCHUS TOP SHELL}

telescopium

telescope

Tranquebar

\section{MUREX}

ROCK SHELL

cbenius

deep black

Friendly Islands

fuscatus

prickley

Senegal

obcliscus

Chinese abelisk

Guadaloupe

asper

rough grained

Isle of France

lincatus

lined

Pacific

vertagus

curve-beak

Amboyna

vertagusvar. B. banded curve-bcak Coromandel 


\begin{tabular}{|c|c|c|c|}
\hline Modern Name & Old Name & English Name & Locality \\
\hline 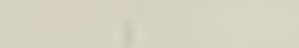 & STROMBUS & SCREW & \\
\hline 1586-1587 tuberculatus & tubcrculatus & tuberculated & Mediterranean \\
\hline 1588 zonatus & zonatus & bclted & Unknown \\
\hline 1589-1591 zonale & zonale & & Antilles \\
\hline PLEUROTOMA & MUREX & ROCK SHELL & \\
\hline 1592 muricata & muricata & prickley & Unknown \\
\hline 1593-1594 flavidula & flavidula & yellowish & Red Sea \\
\hline 1595-1596 virgo & virgo & corded & Unknown \\
\hline 1597-1599 Babylonius & Babylonius & tower of Babel & Amboyna \\
\hline 1600 tigerina & tigcrina & tiger & Unknown \\
\hline 1601 tornatus & tornatus & white tower & Tranguebar \\
\hline 1602 undifera & javanus & javan & Unknown \\
\hline 1603 crispa & crispus & & Unknown \\
\hline \multicolumn{4}{|l|}{$1604-1605$ (see notes) } \\
\hline \multicolumn{4}{|l|}{ TURBINELLA } \\
\hline 1606-1607 polygona & polygonus & many angled & Isle of France \\
\hline \multirow[t]{2}{*}{$1608-1609$ seolymus } & scolymus & flesh colored & Indian Ocean \\
\hline & VOLUTA & VOLUTE & \\
\hline 1610 rapa & & & Indian Occan \\
\hline $161 \mathrm{l}-1613$ gravis & gravis & heavy & Straits of Malacca \\
\hline 1614 pugillarius & muricata & & Antilles \\
\hline 1615-1619 cornigera & tubinellus & turbinated & Amboyna \\
\hline 1620 -1621 rhinoceros & rhinoceros & rhinoceros & New Guinea \\
\hline 1622 globulus & globosa & globose & Unknown \\
\hline 1623 cingulifera & nassa & ribbed & St. Croix \\
\hline
\end{tabular}




\section{5}

\begin{tabular}{|c|c|c|c|}
\hline Modern Name & Old Name & Inglish Name & Locality \\
\hline 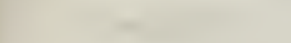 & BUCCINUM & WHELK & \\
\hline $1624-1625$ rustica & rusticumi & emerald & Senegal \\
\hline$=$ & MUREX & ROCK SHELL & \\
\hline $1626-1627$ rigida & rigidus & rigid & Unknown \\
\hline 1628 accuminata & aceuminatus & sharp pointed & Unknown \\
\hline CANCELLARIA & VOLTTA & VOLUTE & \\
\hline 1629-1631 reticulata & reticulata & reticulated & South Sea \\
\hline FASCIOLARIA & MUREX & ROCK SHELL & \\
\hline 1632 -aurantium & aurantium & orange & Unknown \\
\hline $1633-1641$ tulipa & tulipa & tulip & Florida \\
\hline 1642 do. (uniforn & brown) tulipa var. & tulip & Florida \\
\hline $1643-1645$ traperium & trapezium & striped tower & China \\
\hline $1646-1648$ ditto, var. & do., var. a. & do. narrow var. & China \\
\hline
\end{tabular}

FUSUS

\begin{tabular}{|c|c|c|c|}
\hline I649-1650 colossus & colossus & great & Indian Ocean \\
\hline 1651-165̆4 colus & colus & spindle & Indian Ocean \\
\hline $1655-1658$ tuberculatus & colus var. & knobbed spindle & Indian Ocean \\
\hline 1659 Nicobaricus & nicobaricus & Nicobar & Indian Ocean \\
\hline \multicolumn{4}{|l|}{1660 (sce notes) } \\
\hline I661-I663 coronatus & morio & lielmet & Magdalen Isłands \\
\hline 1664 antiqus & antiqus & worn & Dublin Bay \\
\hline $1665-1666$ Nifat & pusio & wreath & Senegal \\
\hline $1667-1669$ corona & corona & crowned & Gulf of Mexico \\
\hline $1670-1673$ articulatus & articulatus & jointed & Unknown \\
\hline \multicolumn{4}{|l|}{ PYRULA } \\
\hline $1674-1675$ canaliculata & eanaliculata & chanelled & $\begin{array}{l}\text { Canarla } \\
\text { I }\end{array}$ \\
\hline
\end{tabular}




\begin{tabular}{|c|c|c|c|}
\hline Modern Name & Old Name & English Name & Locality \\
\hline $1676-1677$ carica & carica & keeled & Unknown \\
\hline $1678-1679$ vespertilio & vespertilio & bat & Tranquebar \\
\hline $1680-1690$ melongena & melongena & open mouthed & Amboyna \\
\hline \multirow[t]{2}{*}{$1691-1692$ perversa } & perversa & reversed & West Indies \\
\hline & BULLA & DIPPER & \\
\hline 1693 reticulata & reticulata & latticed & Indian Ocean \\
\hline $1694-1696$ ficus & ficus & fig & Moluccas \\
\hline \multirow[t]{2}{*}{$1697-1698$ ficoides } & ficoides & fig-like & Indian Ocean \\
\hline & MUREX & & \\
\hline 1699.1710 spierrillus & spicrillus & blunt-lipped & Nalabar \\
\hline \multirow{2}{*}{1711 ternata } & ternatus & ternate & Moluccas \\
\hline & BUCCINUM & WHELK & \\
\hline \multirow[t]{2}{*}{$1712-1713$ bezoar } & bezoar & bezoar & China \\
\hline & MUREX & ROCK SHELL & \\
\hline \multirow[t]{2}{*}{$1714-1719$ rapa } & bulbosum & bulbous & Tranquebar \\
\hline & BUCCINUM & WHELK & \\
\hline 1720 papyracea & rapa & paper & Norway \\
\hline 1721 galeodes & galeodes & & Unknown \\
\hline 1722 angulata & angulatum & angular & Red Sea \\
\hline 1723 citrina & pyrum & pear-shaped & Red Sea \\
\hline \multicolumn{4}{|c|}{ STRUTHIOLARIA MUREX } \\
\hline $1724-1726$ nodulosa & straminins & straw-colored & New Zealand \\
\hline 1727 crenularis & crenularis & crenulated & Unknown \\
\hline \multicolumn{4}{|l|}{ RANELLA } \\
\hline 1728-1730 Argus & Argus & Argus & Indian Oeean \\
\hline 1731-1735 crumena & rasa & frog & Bencoolen \\
\hline
\end{tabular}




\section{7}

Modern Name

1736 spinosa

1737 bufonia

1738 ranina

1739 bitubercularis

MUREX

1740-1741 brandarius

$1742-1743$ cornutus

1744 erassispina

1745-1746 (see notes)

1747-1749 tenuispina

1750-1759 haustellum

$1760-1763$ ramosus

1764-1766 elongatus

$1767-1769$ palmarosre

$1770-1772$ adustus

1773 rufus

1774 seorpio

$1775-1776$ brassica

$1777-1779$ regius

1780-1788 saxatilis

1789 endiva

1790.1792 radix

1793-1799 (see notes

1798-1801 asperimus

$1802-1803$ pinnatus
Old Name

spinosus

bufonius

gyrinus

bitubercularis

brandarius

short beak-shaped Mediterranean

cornutus

tribulus

horned snipe

Adriatic

thorny woodcock Java

$\begin{array}{lll}\text { tribulus duplicatus } & \text { long spined } & \text { Moluceas } \\ \text { haustcllum } & \text { snipe } & \text { Amboyna } \\ \text { ramosus } & \text { branched } & \text { Martiniquc } \\ \text { clongatus } & \text { lengthened } & \text { Indian Ocean } \\ \text { ramosus, var. } & \text { rose bush } & \text { Indian Occart } \\ \text { adustus } & \text { celery } & \text { Bornco } \\ \text { rufus } & \text { red } & \text { Mediterrancan } \\ \text { scorpio } & \text { skcleion } & \text { Moluccas } \\ \text { brassica } & \text { cabbage } & \text { lndian Occan } \\ \text { regius } & \text { royal } & \text { South Sca } \\ \text { saxatilis } & \text { endive } & \text { Phillipine lslands } \\ \text { cichcorrcum } & \text { chickery } & \text { Indian Ocean } \\ \text { radix } & \text { black cdged } & \text { Acapulco } \\ \text { pomum } & \text { apple } & \text { Atlantic } \\ \text { pinnatus } & \text { winged } & \text { Unknown }\end{array}$

\section{Locality}

Tranquebar

Indian Ocean

Mediterrancan

Unknown 


\begin{tabular}{|c|c|c|c|}
\hline Modern Name & otd Name & Engusin IJame & Locatisy \\
\hline $1804-1805$ erinaceus & erinaces & roligh ridge & Dublin Bay \\
\hline 1806 uncinarius' & uncinarius & grappling & Unknown \\
\hline $1807-1808$ gibbosus & gíbbosus & gibbose & Senergal \\
\hline 1809 lamellosus & Iamellosus & lamellar & Falkland Islands \\
\hline 1810 scaber & scaber & rough & Unkiown \\
\hline $1811-1812$ vitulinus & miliaris & scablouls & Nicobar lisands \\
\hline 1813 crispatus & crispatım & curled & King George's Island \\
\hline 1814 trunculus & trunculıs & Tyrian dye & Mediterranean \\
\hline \multicolumn{4}{|l|}{ TRITON } \\
\hline 1815 rariegata & trítonis & triton & Auliogna \\
\hline 1816 nodifera & nodifera & knobbed & \\
\hline 1817 Australe & Australe & southicm & New IJolland \\
\hline $1818-1820$ lampis & lamplas & granulated & Madigasear \\
\hline 1821 corrugata & corrugnta & w rinkled & \\
\hline 1822 sueeinetux & succinctum & girted & New Holland \\
\hline 1823 pileare & pilcare & noduluus oil jar & Antilles \\
\hline $1829-1 \$ 32$ lotoriun & lotorium & angulated oil jar & New Holland \\
\hline 1833 -1834 femornle & femorale & triangular oil jar & Ceylon \\
\hline 1835-1839 рутит & pyrum & pear & Coromandel \\
\hline IS $40-1843$ clavator & clavator & club-shaped & Ceylon \\
\hline 1844 canaliferum & caudatus & long tailed & Coromandel \\
\hline $1845-1855$ anus & anus & grimace & Siam \\
\hline 1856.1857 clathratum & mulus & mule & Hitoe \\
\hline 1838.1861 subdistortum & subdistortum & false-grimace & New Holland \\
\hline
\end{tabular}


1862-1866 maculosum

$1867-1875$ rubicula

1876-1877 dolarium

1878 undusum

ROSTFLIAARIA

1879-1880 curvirostris

1881-1883 pes-pelecani

PTEROCERA

$1897-1908$ lambis

1896 millepeda

1884 scorpio

1885-1895 chiragra

1896-1914 (see notes)

1915-1916 truncata

STROMBUS

1917-1918 gigas
$1919-1920$ latissima
$1921-1923$ accipitrina
$1924-1928$ tricornis
1929 gallus

1930-1935 lentiginosus

1936 bubonius

1937-1938 ditto, (young)

1939-1940 bituberculatus

1941-1943 auris Dianse maculosum

spotted

Barnco

scorbiculator

violet throated

Red Sea

\section{BUCCINUM}

dolarium

undusum

STROMBUS

fusus

pes-pelicani

lambis

millepeda

scorpius

chiragra

bryonia

gigas

latissima

accipitrina

tricornis

gallus

lentiginosus

fasciatus

do. (young)

bituberculatus

muris Diance narrow belted

undulated

SCREW

spindle

pelican's foot

Arabia Felix

Dublin Bay

spider

Banda

centipedc

Ceylon

scorpion

Java

Devil's claw

Ballda

blunt-tipped China

eagles" wing

Barbadoes

broad

Indian Occan

hawks' wing

Asiatic Ocean

three-horncd Red Sea

plough

Martinique

pink lipped Amboyna

rose banded Goree

do. without lip Goree

two knobbed

Diana's ear C'hina 


\begin{tabular}{|c|c|c|c|}
\hline Modern Name & Old Name & English Name & Locality \\
\hline 1944-1949 pugilis & pugilis & thick spined & West Indies \\
\hline 1950-1951 ditto, var. & pugilis var. & \multicolumn{2}{|c|}{ no spines on the body Jamaica } \\
\hline $1952-1955$ pyrulatus & pyrulatus & pear-shaped & Unknown \\
\hline 1956 ranina & ranina & thin lipped & Unknown \\
\hline 1957-1952 gibberulus & gibberulus & pouter & Isle of France \\
\hline 1963-1966 canarium & canarium & partridge & Fort St. George \\
\hline 1967-1969 lubuanus & luhuanus & Lohoe broad-lip & China \\
\hline 1970-I976 mauritiana & mauritiana & pale lipped & Isle of France \\
\hline $1977-1979$ vittatus & vittatus & ribbon & Red Sea \\
\hline I980-1984 suecinctus & succinctus & giràled & Batavia \\
\hline \multicolumn{4}{|l|}{ 1985-1987 (see notes) } \\
\hline I988 variabilis, var. & variabilis, var. & spot mouthed & Unknown \\
\hline 1989 plicatus & dentatus & toothed & Isle of France \\
\hline 1990.1992 lincatus & lincatus & lined & Indian Ocean \\
\hline I993-1994 tridentatus & samar & tliree-toothed & Amboyna \\
\hline $1995-2001$ urceus & urceus & pitcher & Luzone \\
\hline 2002 foridus & floridus & pale & Indian Ocean \\
\hline \multicolumn{4}{|l|}{ ONISCIA } \\
\hline 2003-2012 oniscus & oniscus & wood-louse & West Indies \\
\hline CASSIDARIA & BUCCINCM & & \\
\hline 2013-2015 echinophora & echinophorum & tuberculated & Adriatic \\
\hline \multicolumn{4}{|l|}{ CASSIS } \\
\hline 2016-2017 flammea & fammeum & triangular casket & Jamaica \\
\hline 2018 cornuta & cornutum & horned & Amboyna \\
\hline 2019 tuberosa & tuberosum & casket & West Indies \\
\hline
\end{tabular}




\section{CYPROECASSIS}

2020-2023 rufa

2025 testiculus

CASSIDEA

2026-2033 glauca

2034-2037 arcola

2038 zebra

2039 decussata

2040-2045 erinaceus

2046 granulosa

2047 pyrum

2048 achatina

2049-2057 erinaceus

\section{RICINULA}

2058-2063 horrida

2064 digitata

2065-2066 clathrata

2067-2072 arachnoides

PURPURA

2073 hœmastoma

2074-2085 (see notcs)

2086 pianospira

2087-2092 lapillus

2093-2097 sertum rufum

testiculus

glaueum

areola

strigata

decussatun

erinaceus

granulatum

pyrum

tubcrosum

erinaceus

MUREX

neritoides

digitatus

clathratus

arahnoides

BUCCINUM red casket

Madagascar

bonnet casket Jamaica

white casket Indian Ocean

small dice Meditcrranean

yellow striped Indian Occan

decussated China

hedge-hog Ceylon

grained

Mediterranean

pear

New Holland

casket

New Holland

\section{ROCK SHELL}

mulberry Coromandel

finger Indian Ocean

gemmed Unknown

spider marked Indian Ocean

\section{WHELK}

hœmastomum

rose lipped

Mediterranean

planospirum

flat-tipped

Unknown

lapillus

purple saining Dublin Bay

scrtum 
MONOCERUS

2098-2099 imbricatutn

2100-2102 cingulatum

2103-2104 crassilabrum

2135-2106 citrinum

CONCIIOLEPAS PATELLA

2107-2109 poruvianus

HARPA

2110-2119 ventricosa

2120-2123 articularis

2124 multicostatum

2125 rosca

2126 nobilis

2127-2131 minor

2132 striata

DOLIUM

2133-2134 ringens

2135-2141 pomum

2142-2144 maculatum

2145-2146 fasciatum

2147 perdix

21482152 galcum

2153-2156 olearium

$2157-2158$ variegatum

monodon
cingulatum
crassilabrum
citrinum

lepas

BUCCINUM

harpa

articularis

costatum

rosea

nobilis

minor

cancellata

ringens

pomum

dolium

sulculosum

perdix

galea

olearium

Chinense scaly spme lip Cape Horn

girdled spine lip Mexico

thick lipped Magellan

ycllow spine lip Unknown

LIMPET

Barnacle

Peru

liarp

Indian Ocean

black \& white jointed Indian Occan

double harp Unknown

rosy harp Indian Ocean

noble hary Unknown

Icsser harp Red Sca

cancellated harp Tranquebar

wry-mouthed tun Unknown

appel tun ludian Ocean

spotted tun Amboyna

flat ribbed tun Coromandel

partridge tun Mediterranean

brown tun Naples

tun East lndies

Chinese tun China 


\section{BUCCINUM}

2159 undatum

2160-2163 papillosum

2164-2166 melanostoma

2167-2175 arcularia

2176-2178 thersites

2179 adspersum

2180 testudineum

2181-2182 reticulatum

2183-2186 macula

2187 affine

EBURNA

2188-2191 glabrata

2192-2194 zeylanica

2195-2197 spirata

2198-2199 areolata

\section{TEREBRA}

2200-2204 maculata

2205 flammea

2206-221 l crenulata

2212-2220 dimidiata

2221-2222 muscaria

2223-2228 subulata

2229-2232 duplicata

$\begin{array}{lll}\text { undatum } & \text { waved } & \text { Dublin Bay } \\ \text { papillosum } & \text { prickley lipped } & \text { Java } \\ \text { mclanostoma } & \text { black lipped } & \text { Unknown } \\ \text { arcularia } & \text { broad lipped } & \text { Isle of France } \\ \text { thersites } & \text { pale olive } & \text { Adriatic } \\ \text { turgitum } & \text { red spot } & \text { New Zealand } \\ \text { testudineum } & \text { tortoise } & \text { New Zealand } \\ \text { reticulatum } & \text { reticulated } & \text { Dublin Bay } \\ \text { macula } & \text { spot lipped } & \text { Dublin Bay } \\ \text { affine } & \text { brown striped } & \text { South Sea }\end{array}$

$\begin{array}{lll}\text { glabratum } & \text { glossy } & \text { Tranquebar } \\ \text { zeylanica } & \text { Ceylonese } & \text { Ceylon } \\ \text { spiratum } & \text { acute spired } & \text { Ceylon } \\ \text { areolatum } & \text { small dice } & \text { China }\end{array}$

maculatum

spotted

Pacific

elongatum

lengthened

India

crenulatum

crenulated

Chira

dimidiatum orange \& whitestriped Moluccas

muscaria

square spot lndia

subulata

awl-slaped China

duplicalum

double whirled Indian Ocean 
2233 myuros

2234-2236 aciculina

2237-2238 (see notes)

COLUMBELLA VOLUTA

2239-225l fulgurans

2252-2255 mercatoria

2256 mendicaria

2257 purctata

2258.2275 (see notes)

MITRA

2276-2277 tringa

2278-2282 episcopalis

2283 papalis

2284-2285 pontificalis

2286-2287 cardinalis

2289-2291 scabriuscula

2292 caffra

2293-2299 vulpecula

2300 granatisa

2301-2302 plicaria

CONOHELIX

2303 marmorata

VOLUTA

$231) 4.2306$ melo

2307 semiarnata

caffra

indica strigilatum

cinereum

rats tail

Asiatic Ocean

steel grey

Amboyna

DOVE SHELL

fulgurans

mercatoria

mendicaria

punctata

lightning

Indian Ocean

mercliant

Atlantic

black \& white

spottel

lndian Ocean

Indian Ocean

tringa
episcopalis
papalis

pontificalis

cardinalis

scabriuscula

vulpecula

granatina

plicaria

marmorata

indica, rar.
Indian

China

sand piper

bishops' mitre

popes' mitre

pontifical mitre

cardinals' hat

rough

Friendly Islands

caffre

Africa

foxy

garnet

folded

black \& white

Unknown

half-armed

¿nknown
Amboyra

Indian Ocean

China 
2308-2313 diadema

2314-2315 cthiopica

2316 ducalis

2317 navicula

2318 eymbium

2319-2320 olla

2321 porcina

2322-2327 scapha

2328-2330 imperialis

2331-2336 vespertilio

2337 pellis-serpentis

2338-2342 nivosa

2343-2348 musica

2349-2351 hœbrca

2352 ancilla

2353 Pacifica

2354 fulminata

2355-2356 lapponica

2357-2359 vexillum

2360-2362 (see notes)

2363 undulata

2364 angulata

2365 fulgitrum

2366-2367 aulica diamema

diadem

India

athiopica

iethiopian crown

Japan

ducalis

navicula

cymbium

olla

porcina

scaplıa

imperialis

vespertilio

vespertilio var.

nivosa

musica .

hœbrca

spectabilis

arabica

rupestris

Iapponica

vexillum

undulata

angulata

fulgitrum

aulicd ducal crown Indian Occan

boat

Guinea

gondola

Senegal

melon

Phillipine Islands

keel-margined Coast of Spain

lightning

Cape of Good Hope

imperial

Straights of Malacea

bat

New Holland

snake's shin Indian Seas

snow storm New Holland

music

Curagoa

Hebrew cliaracters Madagascar

whitish

Straights of Magellan

nodulus

Pacific Ocean

rock

Japan

wave lined

American Occan

orauge flag Indian Ocean

undulated New Holland

cornered Unknown

lightning: Unknown

red clouded ludian Ocean 
MARGINELLA

2368 cœrulescens

2369 longivaricosa

2370-2371 tessellata

2372 interrupta

2373-2377 muscaria

OVULA

2378-2387 ovum

2383-2392 gibbosa

2393-2394 birostris

2395 volva

2396 verrucosa

\section{CYPRCA A}

2397 cervina

2398-2401 exanthema

2402-2405 Argus

2406-2409 testudinaria

2410-2415 mauritiana

2416-2418 mappa

2419-2430 histrio

2431-2438 Arabica

2439-2441 mus

2442-2445 rattus

2416 aurora prunum

longivaricosa

porcellana

interrupta

muscaria

\section{BULLA}

ovum

gibbosa

birostris

volva

verrucosa

CYPREEA

occulata

exanthema

Argus

testudinaria

mauritiana

mappa

histrio

Arabica

mus

rattus

aurantium bluish-grey

Goree

snow spot

red spot

dot lined

dotted

DIPPER

cgg

Amboyna

belted

Brazil

thick lipped shuttle China

weavers shuttle Japan

warty

Amboyna

\section{COWRY}

eyed

Atlantic

spotted

Santa Cruz

Argus

Barneo

tortoise shell

Persian Gulf

blackish-brown Nussatella

map

Amboyna

harliquin

Indian Ocean

Arabic character

mouse

Carthagena

rat.

Africa

orange 


\begin{tabular}{|c|c|c|c|}
\hline Modern Name & Name & English Name & Locality \\
\hline 2447-2461 tigris & tigris . & tiger & Java \\
\hline 2462-2471 tigrina & pantherina & panther & Arabia Felix \\
\hline 2472-2478 talpa & talpa & mole & Guinea \\
\hline 2479-2491 carneola & carneola & violet toothed & Amboyna \\
\hline 2492-2495 lurida & lurida & lurid & Sencgal \\
\hline $2496-2502$ vitellus & vitellus & whitc spotted & Island of St. Oma \\
\hline 2503-2508 caput-serpentis & caput-serpentis & snakes head & Otahiete \\
\hline $2509-2513$ cinerea & cinerea & ash grey & Barbadoes \\
\hline 2514-2519 Isabella & Isabella & yellow & Madagascar \\
\hline 2520 olivacea & errones & olive mottled & East Indies \\
\hline 2521-2522 lota & lota & white & Sicily \\
\hline 2523-2530 felina & felina & kitten & Maldives \\
\hline 2531-2532 caurica & caurica & thick edged & Madagascar \\
\hline 2533-2545 erosa & erosa & bordcred & Bengal \\
\hline $2546-2550$ dracena & derosa & variegated & Amboyna \\
\hline 2551-2552 variolaria & cruenta & ferruginous & Amboyna \\
\hline 2553-2559 adusta & ony $x$ & onyx & Coasts of Asia \\
\hline 2560-2569 occellata & ocellata & eyed & China \\
\hline $2570-2571$ cribraria & cribraria & umbilicated & China \\
\hline \multicolumn{4}{|l|}{2572 (see notes) } \\
\hline 2573-2574 hirundo & hirundo & swallow & Maldives \\
\hline $2575-2576$ undata & ziczic & waved & East Indian Ocean \\
\hline 2577 augustata & augustata & narrow & Unknown \\
\hline 2578 ursellus & ursellus & brown spotted & Unknown \\
\hline $2579-2586$ ascellus & ascellus & 3 banded & Amboyna \\
\hline
\end{tabular}




\begin{tabular}{|c|c|c|c|}
\hline Modern Nane & Old Name & English Name & Locality \\
\hline $2587-2594$ helvola & helrola & star & Maldives \\
\hline 2595-2604 cicercula & ciccreula & Vetch & China \\
\hline 2605-2608 globulus & globulus & globular & Amboyna \\
\hline 2609 margarita & margarita & pearl & Amboyna \\
\hline $2610-2614$ staphylæa & staphylæa & groove backed & East Indics \\
\hline 2615 pustulata & pustulata & pustule & Acapulco \\
\hline 2616-2619 nucleus & nucleus & wrinkled & Island of Nussatella \\
\hline $2620-2627$ annulus & annulus & yellow ringed & Alexandria \\
\hline 2628-2640 moneta & moncta & money & Ethiopia \\
\hline 2641-2650 coccinella & Europiea & European & Dublin Bay \\
\hline 2651-2658 pediculus & sulcata & grooved & Antilles \\
\hline TEREBELLUM. & BULLÁ & DIPPER & \\
\hline 2659-2666 subulatum & terebcllum & subulatc & Indian Ocean \\
\hline OLIVA. & VOLUTA & VOLUTE & \\
\hline 2667-2669 porphyria & porpliyria & porphory & Brazil \\
\hline $2670-2678$ textilinea & textilinea & brocade & Antilles \\
\hline $2679-2683$ erythrostoma & erythrostoma & orange tliroat & CoromandeI \\
\hline 2684-2691 maura & maura & Negress & New Holland \\
\hline 2692_2701 maura, var. & maura, var. & do. yellow var. & New Holland \\
\hline 2702 sepulturalis & sepulturalis & dark & Indian Ocean \\
\hline 2703-2705 Senegalensis & Senegalensis & Senegal & Western Africa \\
\hline $2706-2725$ bicincta & bicincta & two banded & Unknown \\
\hline 2726-2734 cpiscopalis & episcopalis & bishops & Unknown \\
\hline $2735-2745$ ispidula & ispidula & enamelled & Indian Occan \\
\hline $.746-2752$ lugubris & lugubris & dark & Indian Ocean \\
\hline
\end{tabular}




\begin{tabular}{|c|c|c|c|}
\hline Modern Name & Name & English Name & Locally \\
\hline 2753-2755 guttata & cruenta & bloodshot & Amboyua \\
\hline $2756-2766$ carneola & carneola & carnelian & Zanguebar \\
\hline 2767 tesselata & tigrina & Tiger & Eastern Ocean \\
\hline $2768-2770$ candida & candidá & white & Unknown \\
\hline $2771-2763$ seripta & seripta & written & Unknown \\
\hline $2774-2775$ literata & literata & lettered & China \\
\hline 2776 tricolor & tricolor & three colored & Timor \\
\hline 2777 sanguinolenta & sanguinolenta & red spot & Jara \\
\hline 2778 undata & ventricosa & ventricose & Ceylon \\
\hline $2779-2782$ inflata & inflata & inflated & Unknowı \\
\hline $2783-2784$ braziliana & pinguis & Biazilian & Brazil \\
\hline $2785-2797$ utriculus & gibbosa & gibbose & Phillipine Islands \\
\hline 2798-2799 do. (short brov & ariety do. & do. & Do. \\
\hline 2800 -2801 auricularia & auricularıa & ear & Brazil \\
\hline $2802-2805$ subulata & subulata & awl & Java \\
\hline $2806-2807$ acuminata & acuminata & sharp-pointed & Indian Ocean \\
\hline 2808-2813 luteola & luteola & yellowish & Unknown \\
\hline
\end{tabular}

2814-2819 (sec notes)

\section{CONUS}

$2820-2824$ literatus

2825-2827 virgo

2828.2830 eburneus

$2831-2834$ ebræus

2835-2836 Princeps

$2837-2840$ generalis

\section{CONUS}

literatus

virgo

eburneus

ebræus

Princeps

gencralis

\section{CONE}

lettered

Ceylon

rirgin

Afriea

ivory

Indian Ocean

Hebrew character China

chieftain

Indian Ocean

general
Cape of Gool Hope 


\begin{tabular}{|c|c|c|c|}
\hline Modern Name & Old Name & English Name & Locality \\
\hline 2841 -2846 capitaneus & capitaneus & captain & Amboyna \\
\hline 2817 Sumatrensis & leopardus & leopard & Java \\
\hline $2848-2857$ Proteus & spuria & Proteus & Gruadalope \\
\hline $2858-2860$ miles & miles & foot-soldier & China \\
\hline $2861-2872$ tesselatus & tessclatus & mosaic & Madagascar \\
\hline $2873-2879$ augur & augur & augur & Ceylon \\
\hline $2880-2890$ lconinus & leoninus & lion & Mexico \\
\hline \multicolumn{4}{|l|}{ LEPTOCONUS } \\
\hline $2891-2899$ amadis & amadis & amadis & Java \\
\hline \multicolumn{4}{|l|}{ DENDROCONUS } \\
\hline $2900-2905$ striatus & striatus & striated & Moluccas \\
\hline $2906-2912$ betulinus & betulinus & birch bark & Batavia \\
\hline 2913-2917 figulinus & figulinus & fig & China \\
\hline 2918-2921 quercinus & quercinus & oak bark & Friendly lslands \\
\hline $2922-2923$ gubernator & gubernator & pilot & Asiatic Ocean \\
\hline 2924 vexillum & vexillum & banner & Malabar \\
\hline \multicolumn{4}{|l|}{ TEXTILIA } \\
\hline 2925-2931 communis & textilc & tissue & Phillipini \\
\hline $2932-2933$ aulicus & aulicus & red tissue & Amboyna \\
\hline 2934-2937 auratus & auratus & gold brocade & Ceylon \\
\hline 2938 Archicpiscopis & Archipiscopis & Archbishop & East Indian Seas \\
\hline 2939 Canonicus & Canonicus & Canon & East Indian Seas \\
\hline 2940.2942 ammiralis & ammiralis & Admiral & Indian Ocean \\
\hline
\end{tabular}


THELICONUS

2943-2945 nussatella

2946-2947 tcrebcllum

TULIPARIA

2948 nebulosa

2949-2952 geographicus

\section{CORONAXIS}

2953-2954 bandanus

2955-2957 arenosus

2958-2959 marmoreus

$2960-2962$ imperialis

2963 fuscatus

2964-2967 eedonulli

2968 aurantius

PUNTICULUS

2969-2970 pulicarius

2971-2974 obesus

2975-2978 arenatus

2979 sponsalis

2980 Cardinalis

2981-2982 mercator

SPIRULA

2983-2984 Peronii nussatclia

terebra

tulipa

geographicus

tulip

geographer

Spanish Main

Madagascar

$\begin{array}{lll}\text { bandanus } & \text { reticulated } & \text { Moluceas } \\ \text { arachnoides } & \text { spider's web } & \text { Coromandel } \\ \text { marmoreus } & \text { marbled } & \text { Barneo } \\ \text { imperialis } & \text { imperial } & \text { Java } \\ \text { fuscatus } & \text { brown } & \text { New Zealand } \\ \text { cedonulli } & \text { eedonulli } & \text { Caraccas } \\ \text { ccdonulli, var. } & \text { yellow cedonulli } & \text { Phillipine Islands }\end{array}$

pulicarius

flca-bite

Java

obesus

arenatus

sponsalis

eoceineus

mereator

NAUTILUS

spirula obese Ceylon

sandy Bataria

pink spotted Isle of Saints, Gorce

Cardinal Martinique

net-work Cape of Good llope 


\section{Modern Name}

NAUTILUS

2985-2988 pompilius

2989-2990 umbilicatus

ARGONAUTA

2991-2994 argo

2995 tubcrculata

CARINARIA

2996 vitroa
OId Name English Name

pearly

Indian Occan

scrobiculatus

sunk spired

New Guinca

ARGONAUTA PAPER SAILOR

argo

eastern

Corsica

tuberculata

tuberculated

Amboyna

PATELLA

\section{LIMPET}

cristata

glassy

Amboyna 


\section{NO'TES.}

97. A species of Mactra, resembling the M. giganten, but thinner more depressed and more elongated transversely.

98. Resembling the last, but rather more prominent at the beaks, probably the young, or a mere variety of $M$. gigantea.

144. A Tellina, apparently eonfounded with the T. sulphurea; but the beak is greatly shorter, the ligament is larger and the transverse striæ deeper and sharper; the colour is the same in both.

179-186. From 179 to 181 , a very much rounded Tellina of white colour, ligament very large, inflexion of the beak small transverse strise irregular, fine length and breadth about 3 inches.-182. A minutely decussated shell, with longitudinal and transverse stria, colour white, yellowish at the beak; ligament moderate; pit very large, smooth; length and breadtl about 1 inch. It has a truncated appearance-183. A very thin slightly-beaked Tellen of a pale-flesh colour, distinetly rayed with white.

252_258. From 252 to 254 is a species nearly allied to C. morphina, differing principally in the colouring; the longitudinal rays being absent; and being slightly more compressed. 256,257 , differ from $\mathrm{C}$. morphina only in being rather paler. 257 and 258 , resemble C. castanca, but are of a paleyellow colonr.

275. Differs from C. chione in being slightly thicker, and baving the cpidermis dull.

294. One species of Cytheria.

309. A very old and rugose specimen of C. flexuosa.

- 373-380. Six species of Venus, the two first resembling V. seripta, but greatly larger, coar'ser, and with stronger transverse strie. 375. A very globose thin shell, much worn, and the characters obseure. 376. A pretty species between V. papilionacea, and V. paphia in appearance. 379. Resembles V. caliste, but the ribs are very thick and smootl. 380. A slightly compressed V. ealiste.

381, 383. Coquille rare et précieuse, surtout lorsqu'on possède les deux valves du mème individu. Lamarl, Anin. Sans. Fert, vol, VI. p. 4.

457, 458. Two species of Cardita. 
172, 475. Coruille singulière prècicuse, recherchée dans les eollections.

400. One valre of a singularly thick Area, resembling $\Lambda$. granosa.

508. Not in Jarnark's eabinet.

563. Cleidotheirus?

573. Distinguished from the other species of Tridaena by the long lollow spines on the ribs, and the peculiar truncation of the anterior side.

586. This Hippopus greatly rescmbles a Tridacna squamosa deprived of its scales, having but five ribs, and five intermediate strix, its margin is semicircular and not triangular as in the other species, nor has it a strong promilence in the middle as in the $H$. maeulata; its form, in fact, is precisely that of a much woin T. squamosa, but at the same time possessing the true characters of the genus Hippopus.

607-609. Throc small indistinet species of Modiola.

649. $\Lambda$ specics of Perna, resembling $P$. isognomon, but large, and having the lobe very small, as in the $\Gamma$. ephippium; the linge resembles the latter shell.

650. Coquille extrèmement rare, recherchée, trés précieusc. Lam. Anim. S. V. vol. VI. p. 144.

656,657 . A species of Avicula of a green colour, with white articulated rays.

710, 711 . The two shells bearing these numbers, are probably the same species, they greatly resemble the P. varius, but the ribs are flattened, there is one small rib in the interstices, and the strix on the surface are transverse instcad of longitudinal.

720. A greatly worn valve, with longitudinal strix, and seven ribs, species doubtful.

731. Coquille très-1’are, trés-recherchée dans les collections. Lam. Anim. S. V. vol. VI. $p .190$.

768, 769. Two small undescribed species of Hyalra, from the Nile.

777, 778. Two Chitons, the first a large whitish species, with the lateral triangles tuberculated, the margin scaly variegated with green and white. 'The other is a very beantiful species, the valves are minutely tuberculated, of a chesnut colour, variegated with white; a large white kecl in the centre of each, and the margin of the valves bright blue; the margin is naked coriaceus yellow.

801. Patella measuring nearly eight inches in length.

839-855. Patellie, the species of which have not been ascertained.

866,867 . Two species of Fissurella, the first very large, but obsenred by parasites; the second small and worn, species doubtful.

879-881. Two species of Calypirax, one white inside, with long tuhular spines; the other, dark chessut within, and having estremaly small spines. 
918, 966. Nine speries of Helix, some of them rare.

988_993. Fonl species of Helix, large.

995-997. Two species of Zonites.

1008_1010. A carinated, dark brown Helix.

1030. A shell so rare that it is quoted by Lamark as only existing in the cabinet of MIr. M. Mr. Valenciennes and Salé.

1050-1061. Seven speeies of Bulimus, some of them execssively rarc.

1082. Coquille très-rare, précieuse, Lam. Anim. S, V. vol. VT. p. 129.

-1141. Coquille rare, recherchée et précieuse, surtout lorsq'elle est bien conservée. Lam. Anim. S. V. vol. VI. p. 159.

_1089-1091. Three species of Aehatina.

1145. One Melania.

1154-1156. Two speeies of Ampullaria, the last number green, witl an orange lip.

1217,1218 . One speeies, of Nerita, large worn.

1222, 1223. Large patulous Natica umbilicus, dark red, divided by a sulcus.

1247-1255. One species of Natica, white, with longitudinal brown stripes.

1271, 1273. Très-belle coqnille, préciense et fort reeherehée dans les colleetions. Lam. Anim. S. V. vol. V.p. 214.

1274, 1275. A large white species of Haliotis. (rubbed.)

1299. One small obscure species of Haliotis.

1347-1354. A Troehns, differing from the T. Niloticus only in the base being very slightly dilated.

1360, 1361. A Trochus, resembling T, inermis, but of great size.

1362, 1363. Coquille grande, rare, précicnse, et fort remarkable. Lam. Anim. S. V. vol. VII.p. 10.

1471-1475. Three species of Monodonta, differing in the disposition of the white markings.

1478-1481. Two speeies of Monodonta, the first white with broad transvcrse red blotches, the last greenish grey with zig-zag black lines, an artieulated black and white band at the sutures.

1530. One large Turbo with the lines of growth sealy.

1555-1557. A large brown mottled specics of Turritella.

1604, 1605 Two species of Pleurotoma, the first with brown spiral ridges; the other having the whorls carinate, and transwerse brown stripes,

1660. A spceies of Fusus rescmbling the F. rigidus, but with the beak as long as the spire.

1745,1746 . The first number is the young of M. crassispina, in all probability; the last differs, in having but two tubereules between each varix. 
1793-1799. Two speeies of Murex, the first resembling M. brasiea, but laving a transverse row of tubereules between the variees; the last two numbers differ in their yellow mouth.

1897-1914. Varieties of Pteroecra ehiragra, (age, colour, monstrosities, \&e.)

1985-1987. Differs from the Strombus suecinetus only in having a keel round the body whorl.

2074-2085. Two species of Purpura, the first having two rows of tubercles on the body whorl, and the lips yellow-the other, different varieties of the P. persiea.

2237-2238. A species of Terelra, rescmbling the $T$, aciculina in shape, but of a white colour, with bluish markings at the sutures.

2360_2362. A species of Voluta, elosely resembling the "orange flag." V. vexillum, but is larger, and has many of the transverse red bands eonneeted by longitudinal red eloudings.

2572. A cowry, differing from the C. olivacea, in being shorter, the margin thieker, and having a square brown spot on the baek.

2814_2819. Four speeies of Oliva, the first resembling O. utrieulus, but is banded and retieulated with dark brown; the next is probably a variety of the o. textilinea. The two next specimens differ from the 0 . seripta ebiefly in size, while the last speeies, resembles 0 . undata in markings, but is muel more slender.
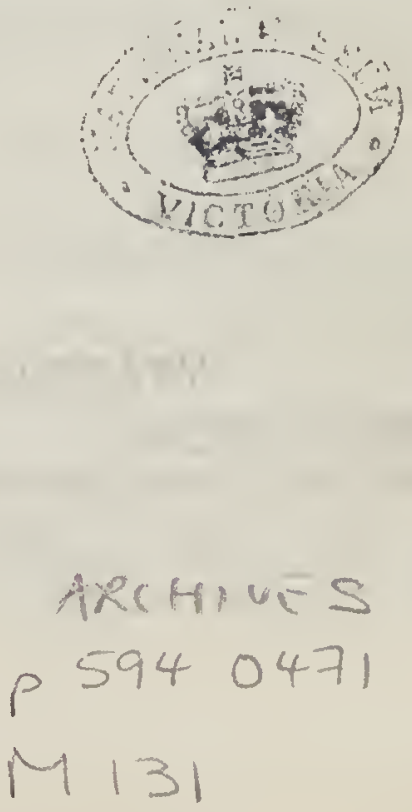

